using pECG-CCTA and rECG-CCTA protocols were $1.7 \pm 0.1 \mathrm{mSv}$ and $3.8 \pm 1.7 \mathrm{mSv}$ respectively. (See table 1 ) None of the studies had coronary images which prevented an accurate assessment of the coronary segments.

Table 1 Comparison of effective radiation dose between pECG-CCTA and rECG-CCTA

\begin{tabular}{|c|c|c|}
\hline \multirow[b]{2}{*}{$\begin{array}{l}\text { Sample } \\
\text { population }\end{array}$} & \multicolumn{2}{|l|}{ Effective radiation dose $(\mathrm{mSv})$} \\
\hline & $\begin{array}{l}\text { Mean dose } \\
\text { reduction (\%) }\end{array}$ & $\begin{array}{l}\text { BMI pECG-CCTA } \\
\text { rECG-CCTA }\end{array}$ \\
\hline $\begin{array}{l}\text { Normal weight } \\
(18.5-<25)\end{array}$ & $1.7 \pm 0.2$ (range, $1.3-2.2 \mathrm{mSv}$ ) & $2.9 \pm 0.9$ (range, $1.5-4.8 \mathrm{mSv}$ ) 41.4 \\
\hline $\begin{array}{l}\text { Over weight } \\
(25-<30)\end{array}$ & $1.7 \pm 0.1$ (range, $1.6-1.7 \mathrm{mSv}$ ) & $3.8 \pm 1.7$ (range, $1.9-5.9 \mathrm{mSv}$ ) 65.3 \\
\hline
\end{tabular}

Conclusions Using a pECG-CCTA protocol as compared to a rECG-CCTA protocol, there was $41.4 \%$ and $65.3 \%$ mean radiation dose reduction for the normal and overweight BMI groups respectively.

\section{Related Subjects: Vacular Medicine e0704 THE STUDY OF EFFECT OF ANGIOTENSIN II ON THE
BIOLOGICAL BEHAVIOUR OF RAT VASCULAR SMOOTH
MUSCLE CELLS IN VITRO}

doi:10.1136/hrt.2010.208967.704

He Guoxiang, Liu Jianping, Jing Tao. Department Of Cardiology, Southwest Hospital, The Third Military Medical University and Chongqing Institute Of Interventional Cardiology, Chongqing, China

Objective To study the effect of Angiotensin II on the roliferation, migration and apoptosis of vascular smooth muscle cell (VSMC) in rats.

Methods The recombinant adenoviral vector, AdCMV-AT2R, containing rat AT2 receptor gene was constructed by homologous recombination, and then it was used to transfer AT2 receptor gene to rat VSMC in vitro. The expression of AT2R mRNA was detected by RT-PCR and the rate of expression in VSMC was determined by flow cytometer. Cell proliferation was determined by incorporation of bromodeoxyuridine (BrdU). The modified Boyden's chamber method was used to test the migration of VSMC. Apoptosis was quantified by flow cytometer.

Results RT-PCR showed that the expression of AT2R mRNA increased obviously in transferred VSMC, and the peak value of expression rate was about $89.51 \%$ at $48 \mathrm{~h}$. When the expression of AT2R was at peak value, the OD value of BrdU incorporation were reduced by $51.6 \%(p<0.01)$, and the number of VSMC migration was also decreased by $62.2 \%(p<0.05)$. The ratio of apoptosis in VSMC was increased from $7.6 \pm 1.6 \%$ in control group to $32.1 \pm 5.5 \%$ in treated group.

Conclusion The results indicated that the expression of AT2R can inhibit the proliferation and migration of rat VSMC and induce its apoptosis.

\section{e0705 COMPARATIVE STUDY OF CARDIO-ANKLE VASCULAR INDEX (CAVI) AND CAROTID INTIMA-MEDIA THICKNESS}

doi:10.1136/hrt.2010.208967.705

Guo Xiaoyu, Li Fangjie. Wang Jing Hospital, China Academy of Chinese Medical Sciences

Objective To contrast measurements by two methods of cardio ankle vascular index (CAVI) and carotid intima-media thickness
(IMT) and discuss the differences and correlation of two methods for clinical application correctly.

Methods The objects are 67 patients with carotid artery ultrasound. We collected the subjects' case history and used VaSera VS-1000 detector to measure CAVI. CAVI $\geq 9.0$ is taken as positive, carotid IMT $>1.0 \mathrm{~mm}$ was positive standards.

Results There are 38 cases with CAVI positive results in all the 67 patients, the positive rate of $56.72 \%$. Carotid IMT was detected in 48 cases positive (accounting for 71.64\%). compared CAVI and carotid IMT with $\chi^{2}$ test, the results showed that $p>0.05$, that is to say the measurements of two methods showed no significant statistical difference. In this data we also respectively observed the influential factors of CAVI and carotid IMT using Logistic multiple regression analysis. The result showed that the main influential factor of CAVI and carotid IMT results was age, while none of clinical diagnosis was enrolled the regression equation.

Conclusions In this data carotid IMT was made as ultrasound index and CAVI was made as the index of pulse wave velocity, we observed two methods validity to early assess atherosclerosis, the results showed that the detection rate of two methods was overall no significant difference, while carotid IMT has a higher trend than CAVI in positive rate to diagnose atherosclerosis. There are difference and correlation between CAVI and IMT. IMT reflects structural changes in arteries, and CAVI are mainly arterial function index, reflecting changes in arterial stiffness. IMT is mainly used to assess specific certain vascular structural changes, CAVI is mainly used to evaluate systemic arterial stiffness. So uniting CAVI and ultrasonic testing could be evaluated vascular morphology and flexibility function simultaneously, which can more fully grasp the extent of vascular lesion. In the influential factors, age is an independent influential factor of the two methods. While hypertension, hyperlipidaemia, and diabetes were not included in regression equation, we considered it might be correlated with the time of clinical diagnosis, smoking and other factors, or may be due to small sample of this study, which need clinical further large sample in-depth study to confirm.

\section{Other: Other}

\section{e0706 EVALUATION OF INTRAVENTRICULAR FLOW IN DCM PATIENTS USING VECTOR FLOW MAPPING}

doi:10.1136/hrt.2010.208967.706

Zhou Xiao, Zhi Guang, Xu Yong, Wang Jing, Tong Kai, Zhao Bei. Pla General Hospital

Aims To assess the change of intraventricular flow due to DCM (Dilated Cardiomyopathy) using VFM (Vector Flow Mapping).

Methods DCM patients and healthy candidates were included into two groups. 3C and 5C images were stored using VFM. Vector velocity of intraventricular flow was measured at the different planes and compared among groups.

Results Six DCM patients and 11 candidates were included. The vector velocity measured at LV outflow tract is lower in DCM group; a flow in direction of LV apex was found in the middle and apical planes of DCM patients while not in healthy candidates; the vector velocity of isovolumic contraction measured at basal plane is found to be higher in healthy group, but lower in the same group at middle and apical planes.

Conclusions In DCM patients, the vector velocity of LVOT is lower, which is accompanied by an abnormal flow distribution in middle and apical parts of left ventricle in both systole and isovolumic contraction. VFM can be used to evaluate the change of the intraventricular blood flow. 